\title{
Die Krise der darstellenden Künste und die Rolle der Kulturpolitik
}

\author{
Annette Zimmer und Birgit Mandel
}

\section{Zusammenfassung}

Aus einer neoinstitutionellen Perspektive werden unterschiedliche Aspekte krisenhafter Entwicklungen in den darstellenden Künsten untersucht. Hierbei liegt der Fokus auf neuen Akteurskonstellationen und den sich daraus ergebenen Herausforderungen an kulturpolitische Prozesse, der Veränderungen der Leitungs- und Steuerungsstrukturen öffentlichgetragener Theater sowie der Infragestellung ihrer Legitimation. Die Beiträge eröffnen neue und komplexe Perspektiven auf veränderte Kontextbedingungen, Handlungsoptionen und Transformationsstrategien in den darstellenden Künsten.

\section{Schlüsselwörter}

Institution Theater • Legitimationsdilemma • Neoinstitutionalismus • Kulturpolitik • Cultural Governance

\footnotetext{
A. Zimmer $(\bowtie)$

Institut für Politikwissenschaft, Westfälische Wilhelms-Universität Münster, Münster, Deutschland

E-Mail: zimmean@uni-muenster.de

B. Mandel

Institut für Kulturpolitik, Universität Hildesheim, Hildesheim, Deutschland E-Mail: mandel@uni-hildesheim.de
} 


\section{$1 \quad$ Krise ist am Theater immer!}

So unisono das Resümee der Kulturschaffenden. Doch die Perspektiven auf Krise und krisenhafte Entwicklungen sind ebenso vielfältig wie deren antizipierte Folgen. Je nach Perspektive des Betrachters/der Betrachterin oder des Betroffenen/der Betroffenen handelt es sich um eine ökonomische, politische, ästhetisch-kulturelle oder aber nachfrage-indizierte Krise, ausgelöst durch eine schwächelnde Wirtschaft, gesellschaftliche Umbrüche oder zurückgehende Besuchszahlen der Veranstaltungen der Theater. Und hiervon gibt es in Deutschland eine beachtliche Anzahl. Gemäß Angaben des Statistischen Amtes des Bundes und der Länder sowie der Theaterstatistik (Destatis 2020; Deutscher Bühnenverein 2019, S. 253) gab es 2018 in Deutschland 142 öffentlich getragene und 199 private Theater (Deutscher Bühnenverein 2019, S. 262), eine Vielzahl freier Theatergruppen- und Einzelkünstler*innen - davon sind allein 2300 im Bundesverband der freien darstellenden Künste (BDFK 2020) -, 128 öffentlich getragene Symphonie- und Kammerorchester (Deutscher Bühnenverein 2019, S. 261), eine beachtliche Zahl von Laien- und semiprofessionellen Theatergruppen und Ensembles - rund 2400 Gruppen mit 100.000 verbandsmäßig organisierten Mitgliedern (Renz und Götzky 2014, S. III) -, mehr als 800 Spielstätten für Theater (Deutscher Bühnenverein 2019, S. 253) sowie - zumindest vor Corona-Zeiten - eine kontinuierlich zunehmende Anzahl von Festivals für Sprech- und Musiktheater sowie auch eine wachsende Zahl von Festivals und Wettbewerben speziell für den künstlerischen Nachwuchs (Deutscher Bühnenverein 2007, S. 256; 2012, S. 266, 2017, S. 262, 2019, S. 262; Hoesch 2019, S. 2). Die außerordentliche Dichte, Diversität und künstlerische Vielfalt des Theaters in Deutschland werden international immer wieder herausgestellt und wertgeschätzt. Insbesondere die Stadtund Staatstheater haben im internationalen Vergleich aufgrund der für das Theater in Deutschland typischen Kombination aus hauseigener Spielstätte, festem Ensemble, Repertoirebetrieb und beachtlicher öffentlicher Förderung ein herausragendes Alleinstellungsmerkmal. Dieses spezifische Theatersystem ist Ergebnis einer langfristigen Entwicklung, die in die Anfänge der Moderne zurückreicht, durch „Fürstenhof und Bürgergesellschaft“ (Wagner 2009) geprägt wurde, sich immer wieder in der Krise befindet und daher infrage gestellt wird.

Zweifellos ist Theater in Deutschland eine gewachsene Institution im klassischen Sinn des historischen Institutionalismus (Steinmo et al. 1992) wie auch der neo-institutionalistischen Organisationstheorie (Powell und DiMaggio 1991). Aus historischer Perspektive sind pfadabhängige Entwicklungen typisch für Institutionen, die sich stets durch eine gewisse Trägheit und ein Beharrungsvermögen der überkommenen Strukturen, Produkte und Prozesse auszeichnen. Institutionen sind 
in diesem Sinne wie Tanker, die nicht so leicht aus der Spur zu bringen sind und sich gegenüber ad hoc-Veränderungen nicht gerade responsiv verhalten. Aus organisationstheoretischer Sicht sind Institutionen aber gleichzeitig in hohem Maße abhängig von ihrer jeweiligen Umwelt. Hinsichtlich ihrer Strukturen, Prozesse und Produkte wie auch im Hinblick auf ihre Organisationskultur und ihr Marketing müssen sie dem entsprechen, was derzeit ,in“ ist und dem dominanten Trend entspricht. Insofern ist nicht Effizienz der Betriebsabläufe Garant für Organisationsüberleben, sondern Übereinstimmung mit dem dominanten Organisationsund Managementmodell und den diesem Modell inhärenten Werten und Normen. Legitimität im Sinne einer möglichst passgenauen Übereinstimmung mit den ,terms of trade“ der jeweiligen Organisationsumwelt und der hier vertretenen Einflussgrößen bzw. Stakeholder ist der Garant für die Existenzsicherung der betreffenden Organisation. Übertragen auf die Institution Theater bedeutet dies: Legitimität ist die Lebensversicherung der spezifisch deutschen Ausprägung von Theater.

\section{Theater im Legitimationsdilemma als Gegenstand empirischer Forschung}

Allerdings befinden sich die Theater hierzulande seit geraumer Zeit in einem Legitimationsdilemma, da sie sich als gewachsene Institutionen mit langer Tradition durch eine gewisse Trägheit - bzw. organisationstheoretisch gerahmt - durch ein gewisses Maß an ,organizational slack“ (Zucker 1987; Leitner und Meyer 2013) auszeichnen. Gleichzeitig sind die Theater spätestens seit Beginn der 1970er Jahre mit einer extrem turbulenten Umwelt konfrontiert, die seither durch tiefgreifende gesellschaftliche Umbrüche und demografische Veränderungen, einer umfassenden Restrukturierung der öffentlichen Verwaltung, fiskalischen Krisen der kommunalen Haushalte, ausgeprägten kulturell-ästhetischen Veränderungen und nicht zuletzt durch das Mega-Ereignis der deutsch-deutschen Wiedervereinigung gekennzeichnet war. Die Theater befinden sich daher in einer permanenten Ausnahmesituation und sind herausgefordert, sich zwecks Überlebenssicherung den veränderten Kontextbedingungen anzupassen und somit ihren ,organizational slack“ in den Dienst der Legitimationsbeschaffung zu stellen, um die „Krise“ ganz gleich welcher Art und welchen Ursprungs - zu meistern. Wie dies erfolgt, vor welche Herausforderungen dies Theater stellt, welche Strategien sie wählen und welche Erfolge sowie Niederlagen hierbei zu verzeichnen sind, wird unter dem Leitmotiv „Krisengefüge der Künste: Institutionelle Transformationsdynamiken in den darstellenden Künsten der Gegenwart" im Rahmen einer von 
der Deutschen Forschungsgemeinschaft geförderten Forschungsgruppe mit Fokus auf Theater in Deutschland untersucht. Unter der Leitung von Prof. Dr. Christopher Balme wird die Forschungsgruppe von den Theaterwissenschaften der Ludwig-Maximilians-Universität in München koordiniert. ${ }^{1}$

Die Gruppe arbeitet u. a. mit der Zielsetzung der Etablierung einer institutionell orientierten Forschungsperspektive in den Theaterwissenschaften als Ergänzung und Korrektiv der bislang dominanten kulturell-ästhetischen. Zusammengeschlossen sind insgesamt sieben Forschungsprojekte, die unterschiedliche Aspekte der Krise als Infragestellung der gewachsenen Institution Theater in seiner für Deutschland spezifischen Ausprägung untersuchen. Die Forschungsgruppe arbeitet unter der Prämisse, dass Krise in der Regel als Auslöser und Motor von Veränderung wirkt und der jeweilige „organizational slack“ eines organisationalen Gefüges eben nicht nur institutionelle Beharrungskräfte perpetuiert, sondern auch Potenziale einer pfadabhängigen und damit institutionenverträglichen Anpassung an veränderte Umweltbedingungen freisetzt und insofern zur Überwindung organisationaler Blockaden und Stillstände beitragen kann.

Im Sinne einer Wirkungskette wird aus neoinstitutionalistischer Perspektive davon ausgegangen, dass die Krise als Herausforderung und Motor zur Transformation durch externe Einflüsse ausgelöst wird. Es sind veränderte Erwartungen, Anforderungen, Wünsche, Interessen und Maßgaben organisationsrelevanter Einflussfaktoren und Stakeholder, die für das Theater krisenauslösend, -verschärfend oder -befriedend wirken können. Die Relevanz der Politik als Einflussfaktor und Stakeholder der überwiegend mit öffentlichen Mitteln geförderten und zu einem Großteil auch öffentlich getragenen Theater - Sprech- und Musiktheater, Freie Szene sowie Festivals - ist in Deutschland als hoch einzuschätzen. Auch wenn Kulturpolitik als Gegenstand politikwissenschaftlicher Analyse in der Regel eher wenig Beachtung erfährt (Zimmer 2019), ist Kulturpolitik in ihrer Bedeutung als Rahmensetzung, Förderung und finanzielle Absicherung wie auch als Garant der Freiheit von Kunst und Kultur für die Theater in Deutschland von eminenter Bedeutung. Dies gilt umso mehr als es sich bei den darstellenden Künsten um den am stärksten geförderten Bereich kultureller Produktion in Deutschland handelt.

Doch Politik und damit auch Kulturpolitik ist in Demokratien mit marktwirtschaftlicher Ordnung ebenfalls in hohem Maße kontextabhängig. Korrektiv und Indikator für die Legitimation sowie Delegitimierung von Politik sind die Stimmabgaben der Bürger*innen am Wahltag. Ob und inwiefern Theater von der Bevölkerung wertgeschätzt, häufig besucht und als meist prominentes, vielfach

\footnotetext{
${ }^{1}$ https://www.krisengefuege.theaterwissenschaft.uni-muenchen.de/index.html
} 
traditionsreiches Bauwerk im Stadtensemble den besonderen Status einer Institution genießt, ist ebenfalls ein wichtiger Einflussfaktor, der sich wiederum sowohl explizit durch Verbesserung oder Verschlechterung der Eigenfinanzierung des Hauses als auch implizit durch die öffentliche Anerkennung politikbeeinflussend auswirken kann. Ferner sind noch die Kulturexpert*innen sowie die Beratungsindustrie anzuführen, die mit ihren Expertisen, Stellungnahmen und Entwicklungsplanungen mehr oder weniger direkt Politik und Verwaltung auch im Kulturund Theaterbereich beeinflussen und ihre Beratung sowie die vorgeschlagenen Modelle wiederum an dominanten Trends der aktuellen Betriebswirtschaftslehre ausrichten. Insofern gestaltet sich das Spektrum der theaterrelevanten Stakeholder als vielfältig und die Kanäle ihrer Einflussnahme auf Theater und Kulturpolitik als komplex. Auf Politik und Verwaltung wirkt jeweils gleich ein ganzes Bündel von Einflussfaktoren ein, wobei Anregungen oder Forderungen, z. B. in Form von Förderprogrammen als distributive Politik oder auch als Restriktionen mittels Etatkürzungen und somit als redistributive Politik, entweder direkt oder gefiltert, an die Theater weitergegeben werden können.

\section{Policy Matters - Kulturpolitik als komplexer Prozess}

Nicht nur im Bereich Theater, sondern insgesamt, sind die Prozesse der Gestaltung und Umsetzung von Politik in jüngster Zeit deutlich komplexer geworden und erfolgen zunehmend unter Mitwirkung vielfältiger Akteure. Politik- und Verwaltungswissenschaft fassen diese Veränderung der Regierungs- und Verwaltungstätigkeit analytisch als Entwicklung von „Government“ zu „Governance“ (Klenk 2019). Modelltheoretisch betrachtet wird unter „Government“ eine hierarchische Steuerung und ein Regieren im Sinne von Entscheidungsfindung unter Ausschluss der Öffentlichkeit sowie ein Verwalten als Top-Down-Umsetzung von Maßnahmen und Direktiven in der Regel durch Ämter und Behörden gefasst. Demgegenüber ist bei einer Regierungs- und Verwaltungstätigkeit nach Maßgabe von „Governance“ der Kreis der beteiligten Akteure nicht auf den engen Zirkel der Politik und Kernverwaltung begrenzt, sondern weiter gefasst und schließt nichtstaatliche Akteure in Form der Vertretung von Stakeholdern der für den betreffenden Politikbereich relevanten Interessen und Akteure mit ein. Diese sind gleichberechtigt und somit ,,auf Augenhöhe“ mit der Politik an politischen Entscheidungsfindungsprozessen beteiligt. Für die Kulturpolitik bedeutet dies insofern eine Herausforderung, als dieser Politikbereich offene Grenzen zu gleich einer ganzen Palette anderer Bereiche wie etwa Bildung, Stadtentwicklung 
und -marketing aufweist und „Kultur“, gerade auch in ästhetisch-künstlerischer Hinsicht, stets kontextabhängig bzw. kontingent ist.

Weitreichende Auswirkungen hat der Übergang von „Government“ zu „Governance" auch für die Umsetzung und Implementation von Politik. Unter dem Leitmotiv des "schlanken Staates“ hat sich seit etwa Anfang der 1990er Jahre ein umfassender Umbau der öffentlichen Verwaltung nach dem Vorbild der Privatwirtschaft und des betriebswirtschaftlich geführten Unternehmens vollzogen. Dieser Umbau des Staates und der öffentlichen Verwaltung, der international unter dem Leitmotiv eines „Reinventing Government“ (Osborn und Gabler 1992) geführt wurde, erfolgte unter Rekurs auf ein komplexes Maßnahmenbündel, das von der Verschlankung und Verbetriebswirtschaftlichung der Kernverwaltung über die Auslagerung von Leistungsbereichen (Outsourcing) und den Einkauf von Leistungen und Diensten unter Wettbewerbsbedingungen (Kontraktmanagement und Competetive Tendering) oder im Rahmen von Kooperationen (Public-PrivatePartnerships) reicht (Jan 2019).

Für die darstellenden Künste in Deutschland hatten diese Veränderungen tiefgreifende Folgen. Die öffentlich getragenen Theater wurden aus der staatlichen Administration ausgegliedert und weitgehend verselbständigt. Gleichzeitig verloren sie infolge der zunehmenden Akzeptanz alternativer Angebote in den darstellenden Künsten - z. B. der Freien Szene - ihren Sonderstatus als die Kulturinstitution. Bisher hatte dies trotz heftiger Debatten keine Schließung von Häusern zur Folge. Auch die im internationalen Vergleich großzügige öffentliche Förderung der Theater, insbesondere der Staats- und Stadttheater, wird bisher nicht grundsätzlich infrage gestellt. Weder von der Politik noch von der Stadtgesellschaft oder der allgemeinen Öffentlichkeit wird dem Theater prinzipiell die Legitimation entzogen, sondern das Theater und die darstellenden Künste genießen weiterhin eine hohe Wertschätzung, wenngleich über die Höhe sowie die Zielsetzung der Förderung zunehmend kritisch diskutiert wird. Mit der Zunahme der Akteure - Freie Gruppen, Festivalmacher*innen, Ausrichter*innen von Wettbewerben, Sponsor*innen und Stiftungen - hat sich der Kreis der Interessenvertreter*innen und theaterrelevanten Stakeholder erheblich erweitert. Schließlich hatte die Neustrukturierung des Bereichs der darstellenden Künste unter Maßgabe betriebswirtschaftlicher Effizienzkriterien beachtliche Auswirkungen auf die hier Beschäftigten, und zwar sowohl auf der Leitungsund Betriebsebene als auch in den künstlerischen Sparten. Auch in den darstellenden Künsten ist es infolge von Prozessoptimierung und Verschlankung zu einer erheblichen Arbeitsverdichtung und damit in der Regel zur Flexibilisierung der Arbeitsverhältnisse bis hin zu einer beachtlichen Zunahme von Zeit- 
und Honorarverträgen, zu deutlichem Personalabbau und nicht selten zu Gehaltsreduzierung und zur Kürzung von sonstigen Leistungen (z. B. Weihnachtsgeld) gekommen. Infolgedessen hat sich mit Blick auf die Leitungs- und Führungsebene in den darstellenden Künsten eine heftige Debatte zur Reform der Corporate Governance der Theater mit der Zielsetzung einer stärkeren Berücksichtigung von Diversity und partizipativeren Führungsstrukturen entwickelt (Schmidt 2019). Im Zuge dessen gewann die „\#MeToo-Debatte“ über strukturellen Machtmissbrauch im Kulturbereich auch in Deutschland deutlich an Virulenz. Auf die veränderten Arbeitsverhältnisse an den öffentlich getragenen Theatern sowie auf die zunehmende Prekarisierung in den darstellenden Künsten wurde mit ,Voice“ (Hirschmann 1970) bzw. mit der Bildung von neuen Interessenvertretungen, wie etwa ,,art but fair", ,ensemble-netzwerk" und ,dancersconnect“, reagiert. Das Tableau der politikrelevanten Themen, Debatten und Diskurse sowie der Interessen- und Anspruchsgruppen hat sich in den darstellenden Künsten in den letzten Jahrzehnten erheblich erweitert. Allerdings hat sich die Kulturpolitik auf diese Veränderungen bisher nur bedingt eingestellt. Der Nachholbedarf ist hier überdeutlich.

Aus politik- und verwaltungswissenschaftlicher Perspektive wird versucht, den veränderten Bedingungen und der gesteigerten Komplexität von Politikgestaltung und Umsetzung im Bereich Kultur unter dem Leitmotiv „Cultural Governance“ als Forschungsperspektive wie als methodisch-analytisches Tool gerecht zu werden (Knoblich und Scheytt 2009; Winter 2019). Die DFG-Forschungsgruppe „Krisengefüge der Künste: Institutionelle Transformationsdynamiken in den darstellenden Künsten der Gegenwart“" ist dieser neuen Sicht auf Kulturpolitik als komplexer Prozess unter Einbindung multipler Stakeholder verpflichtet. Aus inter- wie z. T. auch transdisziplinärer Perspektive wird in den Forschungsprojekten der Gruppe u. a. analysiert, welche Folgen die Veränderung der politischen Kontextbedingungen im Sinne von Cultural Governance für die darstellenden Künste sowohl auf der verfassten Ebene der Organisationen als auch auf der individuellen Ebene der Theaterschaffenden hat, angefangen bei den öffentlich getragenen Theatern über die Freie Szene bis hin zu den Festivals für den künstlerischen Nachwuchs. Darüber hinaus wird in den Projekten empirisch der Frage nachgegangen, wie, mit welchen Strategien und Handlungsoptionen in den darstellenden Künsten auf die durch Kontextveränderung induzierten Herausforderungen mit der Zielsetzung reagiert wird, die hierdurch infrage gestellte Legitimität der Institution neu zu konstituieren. Es geht jeweils darum, der Krise bzw. der Delegitimation der Institution Theater als Organisation, Verfahren oder Norm zu begegnen und diese Krise sogar als Motor für Transformation und Adaption an veränderte Umweltbedingungen $\mathrm{zu}$ nutzen und dadurch wieder erneut Legitimation 
im veränderten Kontext zu gewinnen. Analysierbar wird dieser Prozess durch das Aufkommen von Rechtfertigungsnarrativen, die eine neue Erzählung - einen Mythos (Meyer und Rowan 1977) - von der Relevanz in gesellschaftlicher, politischer oder wirtschaftlicher Hinsicht der Institution - hier des Theaters - als Legitimationsbegründung offerieren.

Der Band „Cultural Governance. Legitimation und Steuerung in den darstellenden Künsten" vermittelt einen ersten Eindruck darüber, wie sich die Forschungsgruppe der komplexen Thematik des Nexus von Politik und darstellenden Künsten aus einer dem historischen Institutionalismus wie dem organisationssoziologischen Neo-Institutionalismus verpflichteten Cultural Governance Perspektive nähert.

\section{$4 \quad$ Zum Aufbau des Bandes}

Die Beiträge des Bandes basieren im Wesentlichen auf Vorträgen, die auf der zweiten Jahrestagung der Forschergruppe gehalten wurden, die im November 2019 in München unter dem Thema „Kulturpolitische Dynamiken in den darstellenden Künsten: Legitimation, Steuerung, Aushandlungsprozesse“" stattgefunden hat. Die Panels der Tagung adressierten zentrale Aspekte und unterschiedliche Dimensionen von Cultural Governance, die auf Basis der Erkenntnisse aus den überwiegend empirischen Projekten der Forschungsgruppe thematisiert und zur Diskussion gestellt wurden. Ergänzt wurden die Beiträge der Forschungsgruppe durch Referent*innen aus der kulturpolitischen Praxis.

\subsection{Politik für Theater}

Analog zur Panelorganisation der Tagung ist der vorliegende Band in vier Kapitel gegliedert. Zunächst wird unter dem Leitmotiv „Politik für Theater“ im ersten Kapitel aus neoinstitutionalistischer und diskurstheoretischer Perspektive sowie aus Sicht kulturpolitischer Akteure der Nexus von Theater und Politik betrachtet. Im einleitenden Beitrag von Christopher Balme „Legitimationsmythen des deutschen Theaters: eine institutionsgeschichtliche Perspektive" geht es um die grundlegende Frage: Warum und wozu eigentlich Theater? Aus organisationstheoretischer Sicht wird ein Überblick über die verschiedenen Begründungen bzw. Legitimationsmythen von Theater gegeben. Hierbei wird deutlich, dass die „Mythen“ bzw. die Erzählungen von der Notwendigkeit von Theater in hohem Maße kontext-kontingent sind und entsprechend dem politischen Zeitgeist 
auf ökonomische, kulturell-ästhetische, pädagogische aber auch auf ethnischnationalistische oder partizipatorisch-sozialdemokratische Begründungsfigurative rekurrieren. Auch der Beitrag von Max Fuchs „,Theaterpolitik als Kulturpolitik“ fokussiert auf die Begründungen bzw. die Legitimationsnarrative der Notwendigkeit von Theater. Aus diskurstheoretischer Sicht wird vor allem die jüngere deutsche Entwicklung und damit die Anfänge, die Umsetzung und die Folgen der Neuen Kulturpolitik mit ihrer Schwerpunktsetzung auf Partizipation und Bildung beleuchtet. Ergänzt werden die beiden theoretisch-konzeptionellen Tours d'Horizon der verschiedenen Legitimationsnarrative für Theater durch zwei Beiträge kulturpolitischer Akteure, die Optionen wie Restriktionen aktueller Kulturpolitik für Theater thematisierten. Hierbei wird u. a. auch deutlich, auf welche Rechtfertigungsnarrative heute Bezug genommen wird und welche „Mythen“ aktuell von der Politik oder politikrelevanten Stakeholdern bedient werden. Im Interview „Die Theater brauchen eine starke Kulturpolitik, um sich verändern zu können “ beschreibt der geschäftsführende Direktor Marc Grandmontagne die Herausforderungen für den Deutschen Bühnenverein als Arbeitgeberund Interessenverband, zwischen Sicherung bestehender Strukturen und dem Einsatz für Veränderungen des Theatersystems. Voraussetzung für eine Bewältigung der anstehenden Aufgaben ist für ihn eine starke öffentliche Kulturpolitik, die ihrer Verantwortung mit transparenten Ziel- und Strategievorgaben gerecht wird. Das Interview mit der Leitung der Kulturstiftung des Bundes, Kirsten Haß und Hortensia Völckers, ist überschrieben mit „Kulturpolitik durch gezielte Förderung: Die Programme der Kulturstiftung des Bundes“. Besonders eingegangen wird hierbei auf die Förderprogramme der Stiftung mit Theaterbezug, die darauf abzielen, Stadt- und Staatstheater als Institution anschlussfähiger an aktuelle Entwicklungen, angefangen bei Digitalisierung, Diversität und Dritte Orte bis hin zu Interkulturalität, zu machen.

\subsection{Theater im Kampf um Anerkennung - Legitimitätsdiskurse und Legitimationsstrategien}

Im zweiten Kapitel ,Theater im Kampf um Anerkennung - Legitimitätsdiskurse und Legitimationsstrategien" geht es um Legitimation als die zentrale „Währung“ zur Existenzsicherung von Theater. In ihrer Einführung zu den fünf Beiträgen dieses Kapitels stellen Axel Haunschild und Bianca Michaels zunächst den engen Bezug zwischen neoinstitutionalistischen Ansätzen in Politikwissenschaft und Organisationstheorie und dem theoretischen Zugang der DFG-Forschungsgruppe „Krisengefüge der Künste“ heraus. Durch grundlegende 
Kontextveränderungen ergeben sich neue Legitimationsanforderungen; es kommt zu Veränderung von Legitimationsstrategien im Bereich der darstellenden Künste und zur Heterogenisierung der Legitimationsnarrative. Diesen Dynamiken in Form von neuen Legitimationsdiskursen, -strategien und Rechtfertigungsnarrativen wird in den Beiträgen des Kapitels nachgegangen. So behandelt der Beitrag von Sebastian Stauss „Künstlerische Qualitätssicherung und Öffnung für neues Publikum. Berufung und Antritt einer Staatsintendanz als kulturpolitische (Selbst-)Legitimation am Beispiel Hannover" einen Legitimationsprozess mittels Personalpolitik auf der Ebene der Intendanz, der in jeder Hinsicht, angefangen bei der Etablierung einer Doppelspitze über Aspekte der Gendergerechtigkeit bis hin zur Spielplangestaltung, genau dem entspricht, was derzeit ,in“ und in hohem Maße als legitimitätsstiftend erachtet wird. Welchem Legitimitätsdruck sich die Freie Szene ausgesetzt sieht und welche Strategien hier zur Anwendung kommen, um im Dienst der Akquise von Fördergeldern die eigene Reputation nachhaltig zu stärken und sich im Bereich der darstellenden Künste optimal zu positionieren, wird von Silke zum Eschenhoff in ihrem Beitrag „Versprechen auf die Zukunft - Der Zusammenhang zwischen Förderung, Produktionsbedingungen und Theaterästhetik am Beispiel der Freien Szene in Niedersachsen " regionalspezifisch näher beleuchtet. Auch der Beitrag von Anja Quickert nimmt Legitimationsstrategien und Rechtfertigungsnarrative der Freien Szene regionalspezifisch in den Blick. In ihrem Beitrag „Krisendiskurse in der Freien Theaterszene (Berlin): Kunst als soziales Wirksamkeitsversprechen im Spannungsfeld zwischen kulturpolitischer Steuerung und künstlerischer Selbstlegitimierung “ kommt sie zu dem Ergebnis einer Repolitisierung der Freien Szene in Berlin, die zunehmend klassische Positionen linker Sozialkritik besetzt und sich damit neu positioniert und legitimiert. Benjamin Hoesch befasst sich in seinem Beitrag „Nachwuchsförderung als Legitimationsmythos " mit einer relativ neuen Arena der darstellenden Künste, den Nachwuchsfestivals, die er als vielfach genutztes Tool zur Legitimation in Form von Rechtfertigungsnarrativen dechiffriert. Allerdings ist der praktische Nutzen, so sein sarkastisches Fazit, gerade für diejenigen am geringsten, für die diese Festivals veranstaltet werden, nämlich für den künstlerischen Nachwuchs. Der abschließende Beitrag von Bianca Michaels „Spielplangestaltung im Kampf um Anerkennung - Diversifizierung als Legitimationsstrategie“ analysiert vor dem Hintergrund deutschlandweit verbreiteter Entwicklungstendenzen in der Spielplangestaltung öffentlich getragener Theater am Fallbeispiel der Münchner Kammerspiele unter der Intendanz von Matthias Lilienthal, Unterschiede und Diskrepanzen zwischen den jeweiligen Erwartungshaltungen der Stakeholder des Theaters vor Ort einerseits und von überregionalem Feuilleton und Theaterkritik andererseits. Für die Münchner Kammerspiele war der Versuch 
der Neupositionierung und Selbstlegitimierung unter Lilienthal zu viel; die Gefahr einer De-legitimation der (Theater-)Institution Kammerspiele infolge der Neuausrichtung unter Lilienthal erschien relevanten Stakeholdern in München als zu groß und insofern nicht unterstützungswürdig, ganz im Gegensatz zur überregionalen Theaterkritik als zwar relevantem, aber nicht ressourcenstarken Einflussfaktor und Legitimationsbeschaffer.

\subsection{Cultural Governance: Kulturpolitische Steuerung und Theater}

Im dritten Kapitel „Kulturpolitische Steuerung und Theater" geht es um den kultur- sowie insbesondere kommunalpolitischen Kontext, um die Veränderung von Cultural Governance durch Verwaltungsmodernisierung sowie sehr konkret um die Zusammenarbeit wie auch Nichtkooperation zwischen den Aufsichtsgremien der öffentlich getragenen Theater und den Theaterleitungen bzw. Intendant*innen. Die vier Beiträge des Kapitels werden eingeführt durch den Theaterwissenschaftler Gerald Siegmund, der die Aufmerksamkeit auf die sehr unterschiedlichen Möglichkeiten und Verfahren kulturpolitischer Steuerung mit Fokus auf Theater lenkt. So beschreiben Johannes Crückeberg und Moritz Steinhauer in ihrem Beitrag „Kulturpolitische Steuerung. Entwicklung und Praxis am Beispiel öffentlich getragener Theater" unterschiedliche Steuerungsmodi als Folge der Veränderung der Kulturverwaltung von der klassischen Hierarchie über das Neue Steuerungsmodell bis hin zu Cultural Governance. Anhand von Fallbeispielen ausgewählter Stadt- und Staatstheater wird die Komplexität des Steuerungsgeschehens verdeutlicht, sodass keines der steuerungstheoretischen Modelle in „Reinkultur" Anwendung findet, sondern Mischformen der Steuerungsmodi festzustellen sind, die modelltheoretisch zu Typenbildung anregen. Der Beitrag von Julia Glesner „Zwischen Bedingung und Freiheit - Über die Zusammenarbeit zwischen Theaterbetrieben und ihren Aufsichtsgremien " zeigt die eminent wichtige Bedeutung einer gut funktionierenden Zusammenarbeit zwischen den öffentlichen Trägern von Theatern - Politik und Verwaltung - einerseits und der Leitung der Häuser andererseits auf. Die Theaterwissenschaftlerin mit langer Praxiserfahrung weist auf Schwachstellen bzw. Steuerungsdefizite hin und zeigt akteursbezogen Wege aus dem Steuerungsdilemma, insbesondere der kommunalen Kulturpolitik auf. Auf ein solches Steuerungsversagen der kommunalen Kulturpolitik geht der Beitrag von Ulrike Hartung „Kulturbetrieb als Schlangengrube? - Kulturpolitische Steuerung de facto und de jure anhand der, Causa Binder" " ein. Behandelt werden die Hinter- und ggf. auch Abgründe des in den 
Medien sehr prominent behandelten Rauswurfs der Intendantin des Tanztheaters Wuppertal, Adolphe Binder. An diesem Fallbeispiel zeigt sich, dass Kulturpolitik z. T. noch weit entfernt ist von einer transparenten, sachlichen und zukunftsorientierten Cultural Governance. Der Beitrag von Lara Althoff, Jonas Marggraf und Annette Zimmer „Kaltstart: Kulturpolitik und Theater in Ostdeutschland - gestern und heute" geht auf eine Veränderung der Cultural Governance im großen Stil ein. Behandelt und kritisch analysiert wird die Transformation der DDRTheaterlandschaft, und zwar im Hinblick auf Veränderungen der kulturpolitischen Einbettung und Steuerung sowie der Veränderung der Führungs- und Leitungsstrukturen der öffentlich getragenen Theater in Ostdeutschland. Es zeigt sich, dass sowohl hinsichtlich der kulturpolitischen Steuerung als auch mit Blick auf das Management der Häuser die Entwicklung ,,im Osten“ nachhaltige Implikationen für ,den Westen“ hatte.

\subsection{Das (un-)entbehrliche Theater? Die veränderte Rolle der (Stadt-)Theater in einer pluralisierten Stadtgesellschaft}

Im vierten Kapitel des Bandes „Das (un-)entbehrliche Theater? Die veränderte Rolle der (Stadt-)Theater in einer pluralisierten Stadtgesellschaft" wird auf das Staats- und insbesondere das Stadtheater fokussiert, das als traditionsreiche Institution der darstellenden Künste mit Alleinstellungsmerkmal im internationalen Kontext in besonderem Maße von Veränderungen der Cultural Governance betroffen ist und insofern seit Jahren erheblich unter Legitimationsdruck steht. Es wird danach gefragt, welche Aufgaben die Stadt- und Staatstheater in einer sich diversifizierenden Gesellschaft aus Sicht unterschiedlicher Anspruchsgruppen übernehmen und auf welche Weise sich die Theater aktuell positionieren. In der „Einführung“ des Kapitels zeigt Birgit Mandel, wie der Begriff der Stadtgesellschaft im Diskurs über Transformationen der öffentlich getragenen Theater als Synonym für die Adressierung der gesamten Bevölkerung in ihrer Pluralität verwendet wird. Verbunden mit dem Anspruch an die Theater, aktiv zur Bildung von Stadtgesellschaft beizutragen, wird hinterfragt, welche Wertschätzung und Legitimität Theater in der Bevölkerung erfahren. In ihrem Beitrag „Das öffentlich geförderte Theater in der Legitimationskrise und unter Innovationsdruck? Ergebnisse einer repräsentativen Bevölkerungsbefragung " zeigt Birgit Mandel, dass es aktuell einen hohen Konsens darüber gibt, die Stadt- und Staatstheater als öffentliches Gut und Instanz kultureller Bildung mindestens auf bisherigem Niveau weiter staatlich zu unterstützen, auch wenn eine Mehrheit die Angebote selbst nicht nutzt und wenig Interesse am Theater hat. Zugleich zeigt sich bei 
den jüngeren Generationen ein nachlassendes Interesse an der klassischen ,Institution Theater“. In ihrem Beitrag ,Stadt-und Staatstheater in Bewegung? Zur Rolle von öffentlich subventionierten Theatern in der Stadtgesellschaft und den veränderten Erwartungen des Publikums" unternimmt Charlotte Burghardt eine Analyse der Selbsteinschätzungen von Theaterschaffenden zu ihrer Rolle und ihrer Wirkung in der Stadtgesellschaft. Hinterfragt wird das in kulturpolitischen Debatten häufig zitierte Narrativ der geringen Responsivität öffentlich getragener Theater gegenüber Erwartungen und Anforderungen ihres Publikums sowie der Stadtgesellschaft. Auf Basis der Ergebnisse einer empirischen Untersuchung ausgewählter öffentlich getragener Theater wird gezeigt, dass die Theatermacher sich sehr wohl auf die veränderten Kontextbedingungen eingestellt und sich in ihrer Programmgestaltung und Auswahl ästhetischer Formate angepasst haben. $\mathrm{Zu}$ einem entsprechenden Ergebnis kommt auch ein weiteres empirisches Projekt der DFG-Forschungsgruppe. Die Beschäftigten der Stadttheater gehen keineswegs vom Veralten der Institution Stadtheater aus, so die Ergebnisse einer empirischen Befragung und Vollerhebung unter den Theaterschaffenden von sechs Stadtheatern, die in dem Beitrag „Arbeiten am Stadtheater: Passion als Beruf? Ergebnisse einer empirischen Untersuchung an sechs Theatern in NRW und Ostdeutschland“ zusammengefasst sind. Die Autor*innen - Lara Althoff, Eckhard Priller und Annette Zimmer - stellen fest, dass die Beschäftigten am Theater zur jeweiligen Stadtgesellschaft dazugehören, sie aber keineswegs ,,auf Rosen gebettet sind“ und zu den gut bis sehr gut Verdienenden zählen, dafür aber für ihre Passion - Theater - arbeiten und leben. Das weit verbreitete Narrativ vom ,erstarrten Theater“, das in erster Linie mit sich selbst beschäftigt ist, wird gemäß den Ergebnissen der Forschergruppe weder von der Leitungsebene der öffentlich getragenen Theater noch von den Beschäftigten, inklusive des nicht-künstlerischen Personals, geteilt. Hilko Eilts geht in seinem Beitrag „Diversifizierung der Programme der Stadtund Staatstheater als Reaktion auf die veränderte Stadtgesellschaft?" der Frage nach, ob und inwiefern das (Vor-)Urteil und Narrativ vom unbeweglichen und von der Zeit überholten Stadt- und Staatstheater durch die Programmgestaltung der Häuser bestätigt wird. Die Ergebnisse seiner sekundärstatistischen Analysen der Theater- sowie Werkstatistiken des Deutschen Bühnenvereins verdeutlichen, dass die Theater sich durch ihre Programmgestaltung in erheblichem Umfang bemühen, auf veränderte Bedarfe und Erwartungen der Stadtgesellschaft einzugehen. Der Frage, wie sich Theater in ihren Programmen und Formaten gegenüber der Stadtgesellschaft öffnen, u. a. mit Unterstützung der Kulturstiftung des Bundes und ihres Förderprogramms „Heimspiel“", ist Lukas Stempel in seinem Beitrag „Mit der Stadtgesellschaft ins Spiel kommen-Der Fonds ,Heimspiel ' der Kulturstiftung des Bundes " nachgegangen. Der Beitrag basiert auf den Ergebnissen einer in 
Form einer Dokumentenanalyse vorgenommen Auswertung der im Kontext von „Heimspiel“ geförderten Theaterprojekte. Hierbei wurde den Fragen nachgegangen, welche Häuser und Projekte gefördert wurden und in welcher Form Probleme der Stadt und Stadtgesellschaft identifiziert und ästhetisch-künstlerisch sowie partizipativ bearbeitet wurden. Auch dieser Beitrag kommt zu dem Ergebnis, dass die Stadt- und Staatstheater sich in beachtlichem Maße bemühen, Legitimitätseinbußen entgegenzuwirken, sich gegenüber den Erwartungen und Anforderungen unterschiedlicher Provenienz responsiv zu verhalten, ohne jedoch ihre grundlegenden künstlerischen Traditionen und Ansprüche aufzugeben. Kurzgefasst: Die Stadt- und Staatstheater sind schwerfällige Tanker, aber sie bewegen sich doch! Bestätigt wird diese Einschätzung im abschließenden Beitrag von Birgit Mandel „Herausforderungen, Transformationen, Legitimität und der Einfluss von Kulturpolitik aus Sicht von Intendant*innen der Stadt- und Staatstheater in Deutschland. Ergebnisse einer quantitativen Befragung ", der in knapper Form die Ergebnisse einer aktuellen Befragung zusammenfasst. Es wird deutlich, dass die Theaterleitungen auf veränderte Bedarfe und Wünsche der Stadtbevölkerung eingehen und bemüht sind, neue Themen, Formate und Stücke in ihr Programm aufzunehmen. Gleichzeitig wird deutlich, dass sich Kulturpolitik bisher wenig responsiv gegenüber dem Theater erweist.

\section{Literatur}

Bundesverband freie darstellende Künste. 2020. Über uns. https://darstellende-kuenste.de/de/ verband/ueber-uns.html. Zugegriffen: 3. Aug. 2020.

Deutscher Bühnenverein. 2007. Theaterstatistik 2005/2006. Köln: Eigenverlag.

Deutscher Bühnenverein. 2012. Theaterstatistik 2010/2011. Köln: Eigenverlag.

Deutscher Bühnenverein. 2017. Theaterstatistik 2015/2016. Köln: Eigenverlag.

Deutscher Bühnenverein. 2019. Theaterstatistik 2017/2018. Köln: Eigenverlag.

Hirschmann, Albert. 1970. Exit, Voice and Loyalty. Responses to Decline in Firms, Organizations and States. Harvard: Harvard University Press.

Hoesch, Benjamin. 2019. Nachwuchskünstler*innen als Innovationshoffnung. Biografische Krise, ästhetisches Risiko?. DFG-Forschungsgruppe Krisengefüge der Künste. LudwigMaximilians-Universität München. München.https://www.krisengefuege.theaterwissensc haft.uni-muenchen.de/working-papers/wp-2_2019/workingpaper_22019.pdf. Zugegriffen: 3. Aug. 2020.

Jan, Werner. 2019. Neues Steuerungsmodell. In Handbuch zur Verwaltungsreform, Hrsg. S. Veit, C. Reichard, und G. Wewer, 127-138. Wiesbaden: Springer VS.

Klenk, Tanja. 2019. Governance. In Handbuch zur Verwaltungsreform, S. Veit, C. Reichard und G. Wewer, 153-164. Wiesbaden: Springer VS.

Knoblich, T., und O. Scheytt. 2009. Zur Begründung von Cultural Governance. Aus Politik und Zeitgeschichte 8 (2009): 34-40. 
Leitner, J., und M. Meyer. 2013. Organizational Slack and Innovation. In Encyclopedia of Creativity, Invention, Innovation and Entrepreneurship, Hrsg. Ellias Carayannis.

Meyer, J., und B. Rowan. 1977. Institutionalized organizations: Formal structure as Myth and ceremony. The American Journal of Sociology 83 (2): 340-363.

Osborne, D., und T. Gaebler. 1992. Reinventing Government. How the Entrepreneurial Spirit is Transforming the Public Sector. Reading: Addison-Wesley.

Powell, W., und P. DiMaggio, Hrsg. 1991. The New Institutionalism in Organizational Analysis. Chicago: Chicago University Press.

Renz, T., und D. Götzky. 2014. Amateurtheater in Niedersachsen. Universität Hildesheim. https://core.ac.uk/download/pdf/148067787.pdf. Zugegriffen: 3. Aug. 2020.

Schmidt, Thomas. 2019. Macht und Struktur im Theater. Wiesbaden: Springer VS.

Steinmo, S., K. Thelen, und F. Longsteth, Hrsg. 1992. Structuring politics: Historical institutionalism in comparative analysis. Cambridge: Cambridge University Press.

Wagner, Bernd. 2009. Fürstenhof und Bürgergesellschaft: Zur Entstehung, Entwicklung und Legitimation moderner Kulturpolitik. Bonn: Kulturpolitische Gesellschaft.

Winter, Thorben. 2019. Cultural Governance. In Governance, Hrsg. K. Möltgen-Sicking und T. Winter, 187-204. Wiesbaden: Springer VS.

Zimmer, Annette. 2019. Kultur als Politik. DFG-Forschungsgruppe Krisengefüge der Künste. https://www.krisengefuege.theaterwissenschaft.uni-muenchen.de/workingpapers/wp-3_2019/workingpaper_32019.pdf. Zugegriffen: 16. Apr. 2020.

Zucker, Lynne G. 1987. Institutional theories of organizations. Annual Review of Sociology 13: $443-464$.

Open Access Dieses Kapitel wird unter der Creative Commons Namensnennung 4.0 International Lizenz (http://creativecommons.org/licenses/by/4.0/deed.de) veröffentlicht, welche die Nutzung, Vervielfältigung, Bearbeitung, Verbreitung und Wiedergabe in jeglichem Medium und Format erlaubt, sofern Sie den/die ursprünglichen Autor(en) und die Quelle ordnungsgemäß nennen, einen Link zur Creative Commons Lizenz beifügen und angeben, ob Änderungen vorgenommen wurden.

Die in diesem Kapitel enthaltenen Bilder und sonstiges Drittmaterial unterliegen ebenfalls der genannten Creative Commons Lizenz, sofern sich aus der Abbildungslegende nichts anderes ergibt. Sofern das betreffende Material nicht unter der genannten Creative Commons Lizenz steht und die betreffende Handlung nicht nach gesetzlichen Vorschriften erlaubt ist, ist für die oben aufgeführten Weiterverwendungen des Materials die Einwilligung des jeweiligen Rechteinhabers einzuholen.

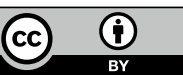

